\title{
Nonlinear optics from off-energy closed orbits
}

\author{
David K. Olsson๑, ${ }^{*}$ Åke Andersson, and Magnus Sjöström \\ MAX IV Laboratory, Lund University, SE-22100 Lund, Sweden
}

(Received 1 June 2020; accepted 22 September 2020; published 19 October 2020)

\begin{abstract}
This paper describes a novel scheme for correcting the second order (chromatic sextupole) magnet lattice and its deployment on a fourth generation multibend achromat electron storage ring. The method is analogous to the well-established linear optics from closed orbits scheme, but uses an off-energy orbit response matrix to characterize the second order optics of a lattice. The matrix is constructed from the difference between two orbit response matrices measured at off-nominal energy, and is approximately linear with chromatic sextupole field strengths. This is utilized in a least squares minimization to find a model which minimizes the difference between the measured and model off-energy orbit response matrix. From this model corrections to the chromatic sextupoles of the machine can be calculated. In effect, for the MAX IV $3 \mathrm{GeV}$ ring, the proposed scheme, NOECO (nonlinear optics from off-energy closed orbits), brings initial sextupole strength variations between achromats in the order of $\pm 8 \%$ down to the order of $\pm 1 \%$. The chromatic machine functions were symmetrized, the measured chromaticities approached the nominal values, and the lifetime of the beam at delivery conditions, with the same level of emittance coupling, increased to $19 \mathrm{~h}$ from $11 \mathrm{~h}$ at previously used sextupole settings.
\end{abstract}

DOI: 10.1103/PhysRevAccelBeams.23.102803

\section{INTRODUCTION}

With the introduction of the fourth generation storage rings the performance of new lattices is being pushed further than ever before. These new machines require careful tuning of the nonlinear magnets in order to achieve the specified lattice momentum acceptance and transverse dynamic acceptance. It is therefore of general interest to find effective correction schemes of nonlinear magnets. Effective in this context means a correction scheme with sufficient precision to achieve the required performance of the nonlinear lattice, and that it is sufficiently fast to not significantly intrude on accelerator study or user experiment time. There are many such potential schemes. A large number of different studies of nonlinear optics are performed at synchrotron facilities around the world. Many of these studies are based on extracting information from turnby-turn beam positional data after the excitation of betatron oscillations. From this the amplitude dependent tune shift, frequency maps, and resonance driving terms can be calculated. These can be used to construct a more accurate model of the nonlinear lattice [1,2], or to optimize the performance of the optics [3]. Although these are powerful

\footnotetext{
*david_k.olsson@maxiv.lu.se
}

Published by the American Physical Society under the terms of the Creative Commons Attribution 4.0 International license. Further distribution of this work must maintain attribution to the author(s) and the published article's title, journal citation, and DOI. methods they may be limited by the decoherence of the turn-by-turn data when measuring on a ring operating at nonzero chromaticity. Since the MAX IV $3 \mathrm{GeV}$ ring operates at a positive chromaticity the decision was made to instead make use of the fact that all sextupoles of the ring are chromatic.

Following the success of the LOCO-scheme [4] (linear optics from closed orbits) in characterizing and correcting the linear magnets of the lattice a similar scheme was developed for characterizing and correcting the chromatic sextupole magnets based on the same principles. The new scheme, named NOECO (nonlinear optics from off-energy closed orbits), is based on the fitting of a lattice model to a measured off-energy orbit response matrix (OEORM), consisting of the difference between two orbit response matrices measured at off-nominal energies symmetrically around the nominal orbit. In this paper the method will be described in detail and needs for modifications compared to the LOCO-scheme will be motivated. The results from an iterative application of this new scheme to the first multibend achromat (MBA) lattice, realized in the MAX IV $3 \mathrm{GeV}$ storage ring [5], will be shown as well as its effect on the dynamic acceptance. The work presented here is a continuation and improvement of the work presented in [6] which in turn was inspired by the work in [7].

The second section of this paper describes the MAX IV $3 \mathrm{GeV}$ storage ring achromat's dipole correctors, beam position monitors (BPMs), sextupoles, and octupoles. In Sec. III a mathematical description of the scheme is presented. In Sec. IV computer simulations of the MAX IV 
$3 \mathrm{GeV}$ ring reveal what parameters must be chosen for this particular lattice, but can also serve as an example of how to apply the scheme to other lattices. Section V presents measurements and the iterative application of the fitting results to the MAX IV $3 \mathrm{GeV}$ storage ring, while Sec. VI presents independent experimental results showing the effects on the dynamic acceptances of the ring.

\section{THE MAX IV 3 GEV STORAGE RING}

The MAX IV $3 \mathrm{GeV}$ storage ring has a seven-bend achromat lattice consisting of 20 optically symmetric achromats. The linear optics of the ring is periodically corrected using the LOCO scheme. A more detailed description of the state of the MAX IV $3 \mathrm{GeV}$ linear optics at the time of writing, as well as further references on the MAX IV $3 \mathrm{GeV}$ ring, can be found in [5].

\section{A. BPMs and dipole correctors}

Each of the MAX IV $3 \mathrm{GeV}$ ring achromats contain 10 BPMs, and 10 horizontal and 9 vertical dipole correctors which can be seen in Fig. 1 [8]. In the case of the vertical correctors the second corrector in Fig. 1 is not present.

The BPM offsets are obtained using the standard quadrupole modulation technique in the Matlab middle layer (MML [9]), the details of which can be found in [5]. The quadrupole modulation used for the offset measurements is achieved with trim coils on adjacent sextupoles in all cases except for the BPMs flanking the straight sections, where the trim coils are placed on octupoles instead. In order to avoid saturation effects the main coils are disabled during offset measurements [5].

All but two BPMs of the ring have a circular geometry and are symmetric in both the horizontal and vertical plane. The first and last BPM in the first achromat are slightly wider in the horizontal plane in order to accommodate for injection. These BPMs have slightly different gain coefficients than the other BPMs of the ring.

\section{B. Sextupoles}

The MAX IV $3 \mathrm{GeV}$ storage ring has a total of five sextupole families, SD, SDend, SFi, SFo, and SFm (see Fig. 2 [8]), all of which are chromatic. The magnets belonging to the same family are connected in series

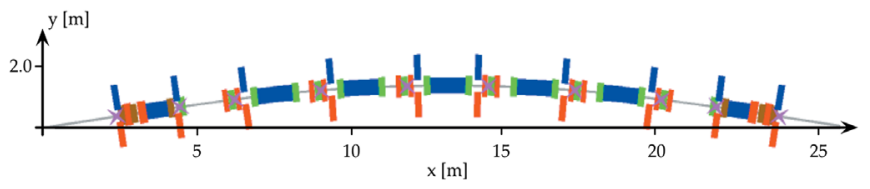

FIG. 1. MAX IV $3 \mathrm{GeV}$ storage ring lattice. Magenta crosses indicate the positions of BPMs, while the blue and orange stripes are the positions of the dipole correctors. In the case of the vertical dipole correctors the second corrector in the image is not present. within each of the 20 achromats. Each circuit of the SD family consists of 10 magnets connected in series, while the remaining families consist of 2 magnets in series. The SFi circuit in achromat 8 is split up for the purpose of BPM offset measurement experiments. In total, the storage ring has 101 independent chromatic sextupole circuits, and no harmonic sextupoles.

\section{Octupoles}

Unlike the sextupoles of the ring, the octupoles are connected in series by family across all achromats. The three octupole families OXX, OXY, and OYY (seen Fig. 2) are predominantly harmonic, with only the OYY family located in a dispersive region.

The octupole settings used throughout this paper were those found through a model-free parameter optimization of sextupole and octupole settings using the RCDS algorithm [10] (courteously provided by Xiaobiao Huang), with the goal of increasing the resilience of the stored beam from a pulsed horizontal kick [11].

\section{METHOD}

Using a measured orbit response matrix to determine and correct the quadrupole gradients is a common approach to correct the linear optics of synchrotrons [4]. A similar approach is used here to correct the strengths of chromatic sextupoles. Below follows a short mathematical motivation and description of the scheme.

At a momentum deviation $\delta$, sextupole fields will give rise to chromatic gradients according to [12]:

$$
k_{\delta, m}(s)=-m(s) \eta(s) \delta
$$

where $m(s)$ is the sextupole field strength and $\eta(s)$ is the dispersion function.

The lowest order perturbation term from quadrupoles and sextupoles experienced by a particle with the momentum deviation $\delta$ is

$$
k_{\delta, \text { total }}=[k(s)-m(s) \eta(s)] \delta
$$

where $k(s)$ is the quadrupole field strength.

The betatron function can be written as an expansion with $\delta$ to first order:

$$
\beta=\beta_{0}+\beta_{1} \delta
$$

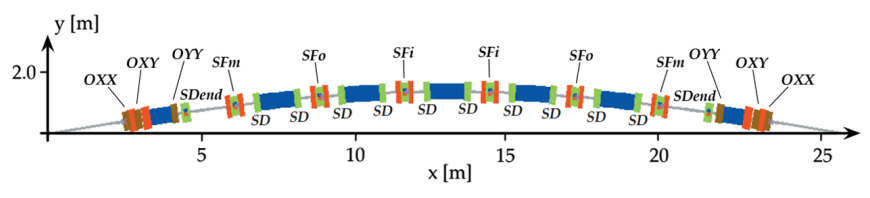

FIG. 2. Locations of the sextupoles and octupoles of the MAX IV $3 \mathrm{GeV}$ storage ring lattice. 
The $\beta_{1}$ function will be referred to as the chromatic functions, the horizontal (vertical) chromatic function is given by [12]. For simplicity, we have excluded the curvature perturbation terms, but they are included in the simulations discussed later in this and the next section (see [13]):

$$
\begin{aligned}
\beta_{1}= & \frac{\mathrm{d} \beta(s)}{\mathrm{d} \delta}={ }_{(-)}^{+} \frac{\beta_{0}(s)}{2 \sin 2 \pi \nu_{0}} \\
& \times \int_{s}^{s+L} \beta_{0}(\zeta)\left(k(\zeta)-m(\zeta) \eta_{x 0}(\zeta)\right) \\
& \times \cos \left[2 \nu_{0}\left(\varphi_{0}(s)-\varphi_{0}(\zeta)+2 \pi\right)\right] \mathrm{d} \zeta
\end{aligned}
$$

Where $\beta_{0}$ is the beta function, $\nu_{0}$ the transverse tune, $\varphi_{0}$ the phase advance, $\eta_{x 0}$ the horizontal dispersion function, and $L$ the circumference of the ring. The above equation holds for either the horizontal or vertical plane. Note that the effect from sextupoles depends on the magnitude of the dispersion at the location of the sextupoles. Harmonic sextupoles do not affect the chromatic functions.

Inserting Eq. (3) into the formula for the oscillatory closed orbit performed by a particle experiencing a dipole kick of magnitude $\theta$, we get:

$$
\begin{aligned}
u(s)= & \sqrt{\left(\beta_{0}(s)+\beta_{1}(s) \delta\right)\left(\beta_{0 \theta}+\beta_{1 \theta} \delta\right)} \theta \\
& \times \sin \left[\nu_{0} \varphi_{0}(s)-\nu_{0} \varphi_{0 \theta}\right]
\end{aligned}
$$

Here $u(s)$ is the transverse displacement of the particle, either horizontally or vertically. The subscript $\theta$ refers to the function evaluated at the longitudinal position of the dipole kick. Expanding and keeping only first order terms of $\delta$ and taking the difference between a kick at a positive and a negative off-energy:

$$
\begin{aligned}
u_{\delta}(s)-u_{-\delta}(s)= & {\left[\sqrt{\frac{\beta_{0}(s)}{\beta_{0 \theta}}} \beta_{1 \theta}+\sqrt{\frac{\beta_{0 \theta}}{\beta_{0}(s)}} \beta_{1}(s)\right] \delta } \\
& \times \theta \sin \left[\nu_{0} \varphi_{0}(s)-\nu_{0} \varphi_{0 \theta}\right]
\end{aligned}
$$

Since $\beta_{0}$ does not depend on $m$ while $\beta_{1}$ is linearly dependent on $m$, the oscillatory closed orbit of an offmomentum particle experiencing a dipole kick is also linearly dependent on $m$. By taking the difference between the closed orbit at $+\delta$ and $-\delta$ the contribution independent of momentum is canceled, leaving only the contribution linearly dependent on the chromatic sextupoles. Higher order effects, such as those from octupoles are not included in this derivation, but will be shown to be negligible in Sec. III A for the specific case of the MAX IV $3 \mathrm{GeV}$ ring. Additionally, the NOECO-scheme is not able to characterize and correct harmonic sextupoles due to them not contributing a chromatic gradient. Since the MAX IV $3 \mathrm{GeV}$ ring only has chromatic sextupoles this will not be an issue in the case of this machine.
Instead of using the orbit response matrix (ORM), as is done in the case of linear optics symmetrization, the 2nd order optics symmtrization uses an off-energy orbit response matrix (OEORM). The OEORM is measured by taking the difference between two ORMs measured at some $\pm \delta$. The sextupole strengths of the model are altered until there is an agreement between the model and measured OEORM. The sextupole values which give the best agreement can then be used to correct the sextupoles of the machine.

The software used for modeling and fitting of the OEORM was Accelerator Toolbox (AT [14]), and Matlab Middle Layer (MML). The ORM at a momentum deviation $\delta$ from nominal is defined as:

$$
\left(\begin{array}{c}
x_{\delta} \\
y_{\delta}
\end{array}\right)=M_{\mathrm{ORM}, \delta}\left(\begin{array}{c}
\theta_{x} \\
\theta_{y}
\end{array}\right)
$$

From this the OEORM is defined as:

$$
M_{\mathrm{OEORM}}=\frac{\left(M_{\mathrm{ORM},+\delta}-M_{\mathrm{ORM},-\delta}\right)}{2 \delta}
$$

or equivalently:

$$
\left(\begin{array}{c}
x_{+\delta}-x_{-\delta} \\
y_{+\delta}-y_{-\delta}
\end{array}\right)=M_{\mathrm{OEORM}} 2 \delta\left(\begin{array}{l}
\theta_{x} \\
\theta_{y}
\end{array}\right)
$$

The fitting of the model OEORM to the measured data was done by a least square minimization with the penalty function:

$$
\begin{aligned}
\chi^{2} & =\sum_{i j} E_{i j}^{2} \\
& =\sum_{i j} \frac{\left(M_{\text {OEORM,meas }, i j}-M_{\text {OEORM,model }, i j}\right)^{2}}{\sigma_{i}^{2}}
\end{aligned}
$$

where the data from each BPM is weighted by the individual BPM noise, $\sigma$.

The minimization was done using a Gauss-Newton method with sextupole strengths, BPM gains, and corrector kicks as fitting parameters. Both the BPM gains and corrector kicks modulate the amplitude of the OEORM signal. In order for the fitting procedure to be able to separate the effect of the BPM gains and the corrector kicks the 2 nd order dispersion, $\eta_{1}$, was added as an extra column to the OEORM. The 2nd order dispersion is defined through the expansion of the dispersion function with respect to a momentum deviation $\delta$ :

$$
\eta=\eta_{0}+\eta_{1} \delta+\mathcal{O}\left(\delta^{2}\right)
$$

The addition $\eta_{1}$ to the fitting procedure is analogous to the use of the 1 st order dispersion of the LOCO fitting 
procedure [4]. The $\eta_{1}$ values will depend linearly on the BPM gains and sextupole strengths, but not on the strength of the corrector kicks. No parameters affecting the coupling were included in the fit, thus the off-diagonal quadrants of the OEORM were not fitted.

\section{A. The Jacobian}

The minimization of $\chi^{2}$ [see Eq. (10)] is done by iteratively solving:

$$
-E_{i j}=\frac{\partial E_{i j}}{\partial K_{l}} \Delta K_{l}
$$

for $\Delta K_{l}$, where $K_{l}$ are the fitting parameters. In order to save time during the fitting procedure a static Jacobian, $\frac{\partial E_{i j}}{\partial K_{l}}$, was used. This Jacobian was calculated around the nominal model lattice.

When performing the fitting two different Jacobians were used. The first was a full sextupole circuit Jacobian, a $(402 \times 380) \times(101+402+380)$ matrix, which allows each sextupole circuit to be fitted individually. Due to the fitting procedure being prone to increasing the setpoints of the SDend and SFm sextupole families against each other a second Jacobian was also used. This Jacobian was a $(402 \times 380) \times(81+402+380)$ matrix in which the SDend and SFm sextupole family circuits were treated as a combined parameter in each achromat. The 402 in the matrix dimensions is the sum of all horizontal and vertical BPM readings, 380 is the sum of all horizontal and vertical dipole corrector magnets, and 101 and 81 are the number of independent sextupole circuits in the case of fully independent circuits and combined SDend-SFm circuits respectively. BPMs which were not in use in the machine, due to, e.g., hardware issues, could be excluded from the fit. Dipole corrector magnets too close to saturation to apply the desired measurement kick were also excluded.

During the fitting procedure the pseudo inverse of the Jacobian was calculated using singular value decomposition (SVD). In the case of either Jacobian, one of the singular values was excluded. The magnitude of the excluded singular values can be seen in Fig. 3. Both of

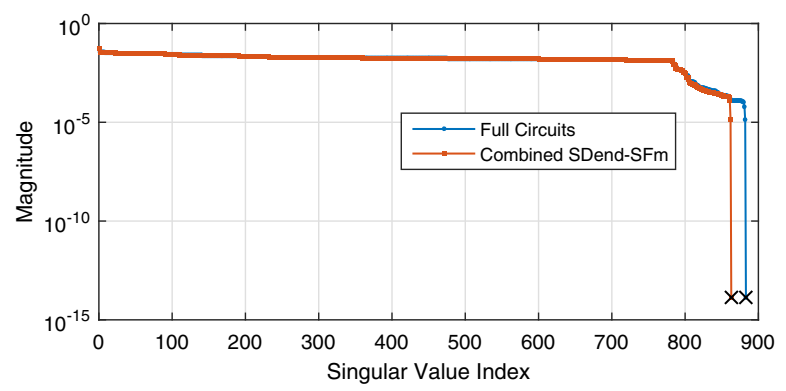

FIG. 3. Singular values of the full sextupole circuit Jacobian and the combined SDend-SFm Jacobian. The marked values were excluded from the fitting procedure.
TABLE I. The effect of each magnet family on the OEORM. The value is calculated by taking the absolute sum of the circuits contribution to the Jacobian. The effect of the octupoles are several orders of magnitude smaller than that of the sextupoles and can therefore safely be excluded from the fitting procedure. The SFi circuits in achromat 8 are treated separately since they each consist of one magnet instead of two.

\begin{tabular}{lcc}
\hline \hline Sextupole family & Absolute sum & Normalized \\
\hline SD & $2.1683 \mathrm{~m}^{3}$ & 1 \\
SDend & $0.2981 \mathrm{~m}^{3}$ & 0.1375 \\
SFi & $0.7733 \mathrm{~m}^{3}$ & 0.3566 \\
SFi (achromat 8) & $0.4436 \mathrm{~m}^{3}$ & 0.2046 \\
SFo & $0.4700 \mathrm{~m}^{3}$ & 0.2167 \\
SFm & $0.4904 \mathrm{~m}^{3}$ & 0.2262 \\
OXX & $4.7470 \times 10^{-5} \mathrm{~m}^{4}$ & $2.1893 \times 10^{-5} \mathrm{~m}$ \\
OXY & $5.1799 \times 10^{-5} \mathrm{~m}^{4}$ & $2.3889 \times 10^{-5} \mathrm{~m}$ \\
OYY & $1.0085 \times 10^{-5} \mathrm{~m}^{4}$ & $4.6512 \times 10^{-6} \mathrm{~m}$ \\
\hline \hline
\end{tabular}

the excluded modes correspond to increasing the vertical BPM gain while decreasing the vertical dipole corrector gain or vice versa. The same singularity exists in the horizontal plane, but can be resolved by including the horizontal 2 nd order dispersion in the fit. In the vertical plane the 2 nd order dispersion is nominally zero and only affected by coupling terms. Since these are not included in the fitting procedure the singularity is not resolved. The excluded modes had no effect on the sextupole circuit settings, which allows them to be excluded without affecting the final sextupole settings of the fit. Further exclusion of singular values will have an effect on the sextupole settings and thus lead to a solution which is less representative of the machine on which the OEORM was measured.

Only sextupoles, BPM gains and dipole corrector magnet kicks were part of the fit. The magnitude of the effect the sextupoles and octupoles of the MAX IV $3 \mathrm{GeV}$ lattice have on the OEORM can be seen in Table I. From the table it is clear that the effect of the octupole magnets is several orders of magnitude smaller than that of the sextupoles. Thus, the octupoles can be safely excluded from the OEORM fitting procedure without affecting the final sextupole settings.

\section{B. The measurement procedure}

The data required for the fitting procedure was measured using the following procedure: (i) The momentum of the beam was shifted by $-\bar{\delta}$ by changing the master rf frequency. ${ }^{1}$ (ii) The dispersion of the energy shifted beam was measured using a bipolar shift of energy around the new beam energy. (iii) An ORM was measured at the new

\footnotetext{
${ }^{1}$ Care has to be taken with regard to the energy dependence of the momentum compaction factor. In the case of the MAX IV $3 \mathrm{GeV}$ ring, simulations have shown this effect to be negligible.
} 
beam energy. (iv) The BPM noise was measured at the new beam energy. ${ }^{2}$ (v) For a bipolar OEORM measurement the above steps were repeated at an equal positive energy shift $\bar{\delta}$ from nominal. (vi) The 2nd order dispersion was constructed by taking the difference between the two measured dispersions and normalizing by $2 \bar{\delta}^{3}$ (vii) The OEORM was constructed by taking the difference between the two measured ORMs and normalizing by $2 \bar{\delta}$.

The BPM noise was measured at both energies as it eliminates a potential source of error without significantly increasing the measurement time.

\section{Correction scheme}

From the model fitted to the measured OEORM a vector of sextupole circuit strengths can be extracted. Using this vector, and the goal values of the circuits, a correction factor for the sextupole settings of the machine at the time of measurement can be calculated according to:

$$
m_{\text {machine,new }}=\frac{m_{\text {goal }}}{m_{\text {fitted }}} m_{\text {machine,old }}
$$

where $m_{\text {machine,old }}$ and $m_{\text {machine,new }}$ are the old and new sextupole settings respectively, $m_{\text {fitted }}$ is the sextupole strengths found by the fit, and $m_{\text {goal }}$ are the goal sextupole settings.

Due to factors such as unknown sextupole error sources being attributed to the sextupole magnets and model discrepancies a single iteration of measuring, fitting, and correction will likely not be sufficient for convergence of the sextupole settings. Instead, the process will need to be iterative: applying the new sextupole settings, and repeating the measurement and fitting procedure from there. The choice was made to cycle the magnets when applying the new sextupole settings in order to not stray from the known measured hysteresis curve.

\section{SIMULATIONS}

\section{A. Linearity of the OEORM with $\bar{\delta}$ and $\theta$}

The linearity of the OEORM with sextupole strength depends on the magnitude of the energy-shift, $\bar{\delta}$, used when measuring the OEORM. In order to find an optimal energyshift the linearity of the OEORM with sextupole strengths was investigated for a number of different energy shifts. For each magnitude of the energy-shift the off-energy orbit response (OEOR) was simulated for 10 equally spaced sextupole strengths, corresponding to $\sim 10 \%$ of the total sextupole range. A linear fit was done to the OEOR

\footnotetext{
${ }^{2}$ This was done by taking the RMS of the BPM beam position over $180 \mathrm{~s}$ after removing the linear component of the drift in position.

${ }^{3}$ In the case of the MAX IV $3 \mathrm{GeV}$ ring 3rd and higher order dispersion is a negligible contribution to this measurement.
}

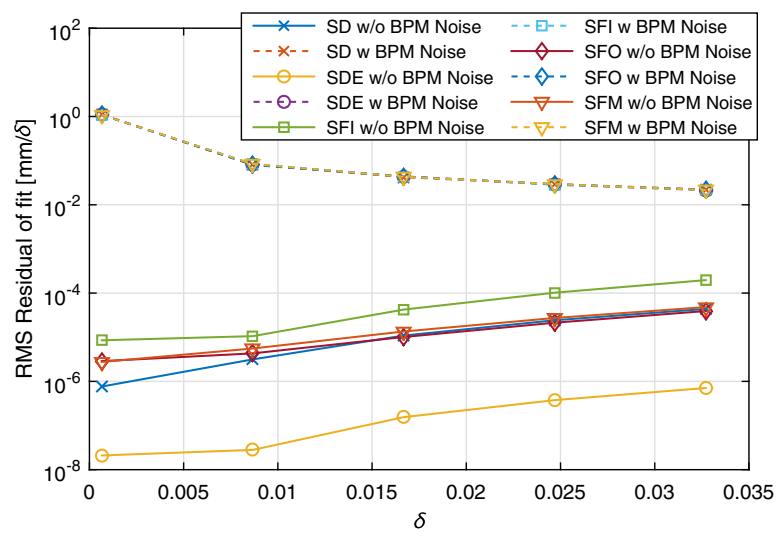

FIG. 4. RMS residuals of a linear fit to the OEOR dependence on a $\sim 10 \%$ sextupole change vs used energy-shift. The simulated BPM noise is normally distributed with $\sigma=0.40 \mu \mathrm{m}$. The nonlinear contribution of the BPM noise dominates, making it beneficial to choose as large an energy-shift as possible.

dependence on sextupole settings, the residual of which can be seen in Fig. 4. The smaller this residual is the more linear with sextupole strengths is the OEORM at that particular energy-shift. When adding a normally distributed BPM noise of $\sigma=0.4 \mu \mathrm{m}$, corresponding to the BPM noise at $3 \mathrm{~mA}, 3 \mathrm{~Hz} \mathrm{BW}$, it becomes clear that the nonlinear contribution from the increase in energy-shift is drowned out by the BPM noise. For example, at $\bar{\delta} \approx 2.5 \%$ the RMS residual for the SFi family of sextupoles is $2.5 \mathrm{~nm}$ compared to the residual of $0.4 \mu \mathrm{m}$ from the BPM noise. In order to decrease the effect of the BPM noise, and thus increasing the linearity of the OEORM, the energy-shift should be as large as possible while still allowing an ORM measurement.

The same investigation was performed for the dipole corrector delta, $\theta$, used when measuring the OEORM. It was found that the nonlinear contribution from the increase of $\theta$ was several orders of magnitude smaller than the contribution from BPM noise, within the range of available $\theta$ s. Additionally, an increase of $\theta$ leads to a greater decrease of the BPM noise contribution than the increase from nonlinearities. In order to minimize the RMS residual of the fit the corrector delta should be as large as possible. However, this would exclude many correctors due to saturation. A compromise had to be found, where the dipole corrector modulation is sufficiently large without saturating too many correctors.

\section{B. Optics characterization}

The NOECO scheme's ability to extract the 2nd order optics from a measured OEORM was tested by simulating the OEORM of a lattice with certain errors, and then fitting the lattice model to the simulated OEORM. The simulations were run in AT and MML. This environment was the same environment used when fitting measurements of the machine. How accurately the fit was able to recreate 


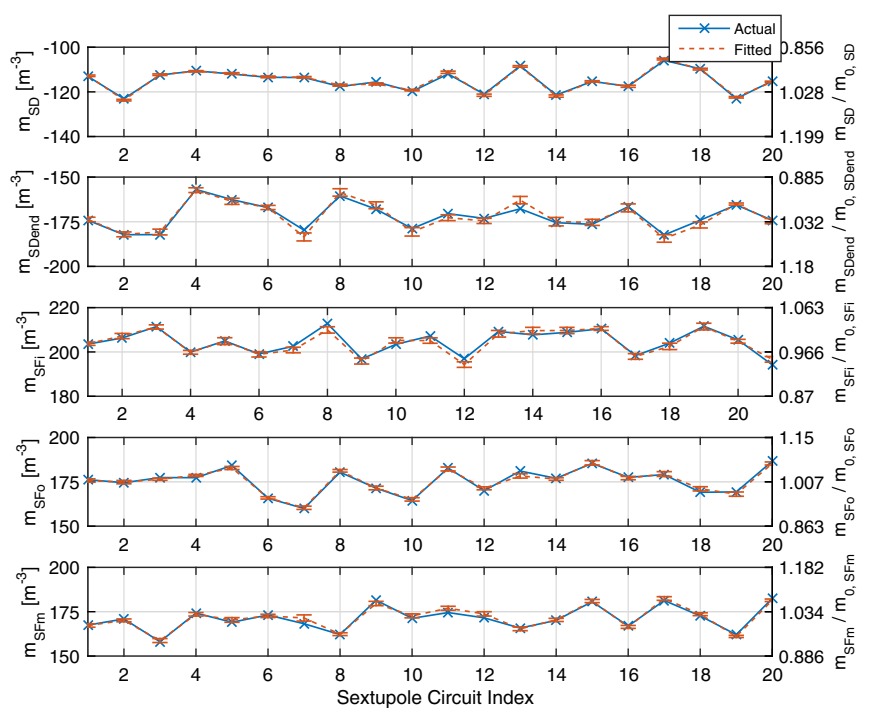

FIG. 5. Introduced and found sextupole circuit errors from one simulation seed. The fitting procedure is able to accurately identify the sextupole settings. The error bars are the standard deviation of 10 error seeds.
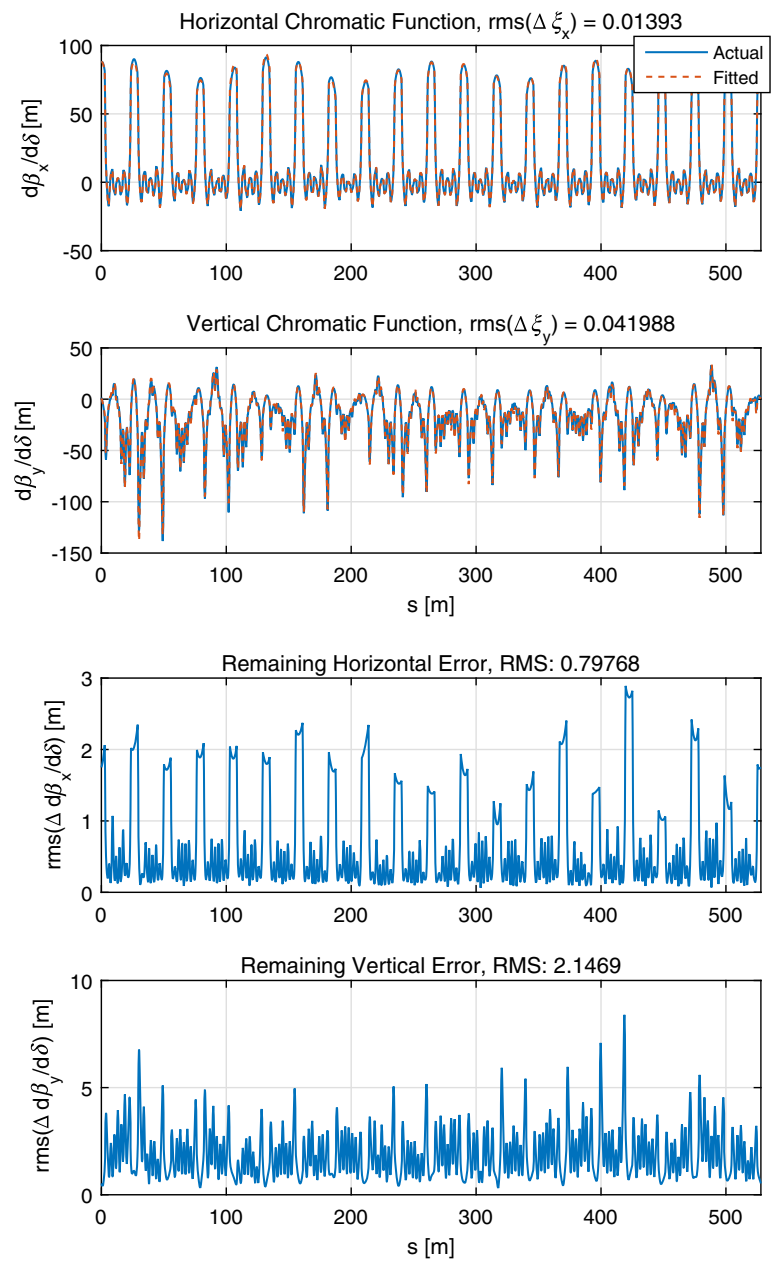

FIG. 6. The chromatic functions of one seed with sextupole circuit errors and the fitted model (top), and the RMS remaining error calculated from 10 error seeds (bottom).
TABLE II. RMS of the chromaticity and chromatic function error of the 10 error seeds.

\begin{tabular}{lcc}
\hline \hline & Circuit errors & Magnet errors \\
\hline$r m s\left(\Delta \xi_{x}\right)$ & 0.0139 & 0.0175 \\
$r m s\left(\Delta \xi_{y}\right)$ & 0.0420 & 0.3640 \\
$r m s\left(\Delta \beta_{x, 1}\right)[\mathrm{m}]$ & 0.7977 & 2.0391 \\
$r m s\left(\Delta \beta_{y, 1}\right)[\mathrm{m}]$ & 2.1469 & 4.7110 \\
\hline \hline
\end{tabular}

the simulated lattice with errors was determined by looking at the agreement of the chromatic functions.

Since sextupole errors from other sources, e.g., misaligned octupoles, will be attributed to the sextupole magnets the fitting procedure needs a few iterations to converge. The nonlinear component of the MAX IV $3 \mathrm{GeV}$ storage ring OEORM with regard to sextupole circuit strength was found to be relatively small (see Secs. IVA). As a result, the Jacobian is relatively unchanged with sextupole settings, making the static Jacobian a decent approximation when fitting, with the only sacrifice being slower convergence.

\section{Sextupole errors by circuit}

Initial simulation tests were performed by introducing sextupole errors by circuit to the nominal lattice. The errors were normally distributed with $\sigma=4 \%$, and a cutoff at $2 \sigma$. A normally distributed transversal alignment error was added to all magnets with $\sigma=25 \mu \mathrm{m}$ and a cutoff at $2 \sigma$, and a normally distributed BPM noise with $\sigma=0.40 \mu \mathrm{m}$ was added to the simulated OEORM to represent the BPM noise at a beam current of $3 \mathrm{~mA}$. The simulation was run for

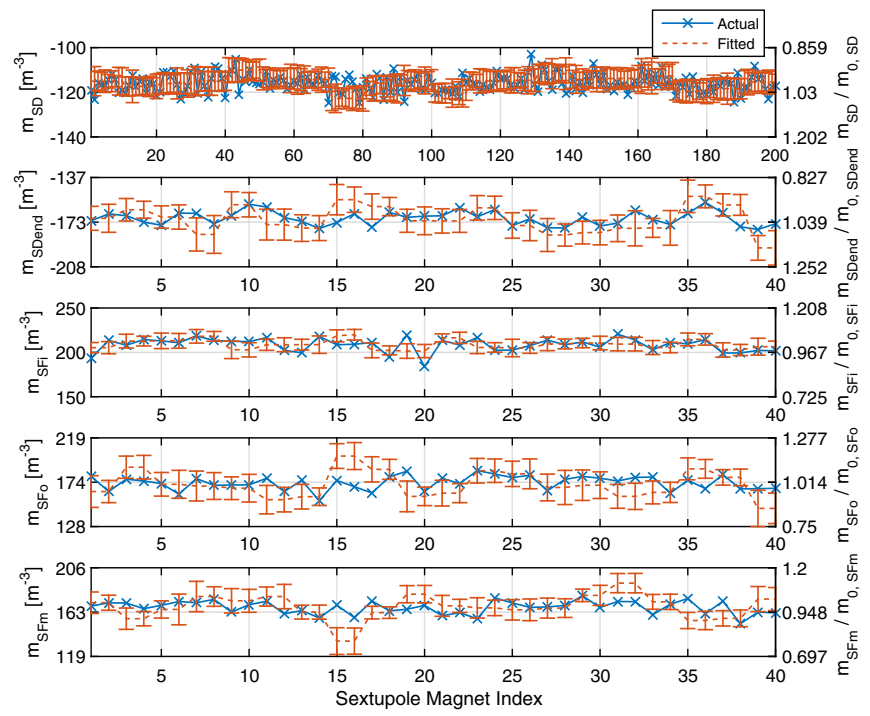

FIG. 7. Introduced sextupole magnet errors and found sextupole circuit settings from a single error seed. The error bars are the standard deviation of 10 error seeds. The found settings do not directly correspond to the average magnet errors in a circuit, despite this the fit is able to reproduce the chromatic functions (see Fig. 8). 
10 different error seeds. For each seed the model was fitted to the simulated OEORM with the strength of all sextupole circuits, BPM gains, and dipole corrector magnet kick strengths as fitting parameters. The OEORMs were simulated at a bipolar energy-shift of $\bar{\delta} \approx 0.16 \%$ from nominal, which proved to give sufficient signal-to-noise ratio. Each case was fitted for 5 iterations which was enough for good convergence. In Fig. 5 the introduced and identified sextupole strengths of a single error seed can be seen, along with an error-bar calculated from all 10 error seeds. The method can clearly identify the sextupole circuit errors.

The fit was also able to recreate the initial model chromatic functions as well as the chromaticities with a high degree of accuracy (see Fig. 6 and Table II).

\section{Sextupole errors by magnet}

The sextupole circuit errors represent a power supply outputting the wrong current. The machine might also have circuit independent sextupole errors, e.g., from a partially short-circuited magnet or manufacturing errors. These were
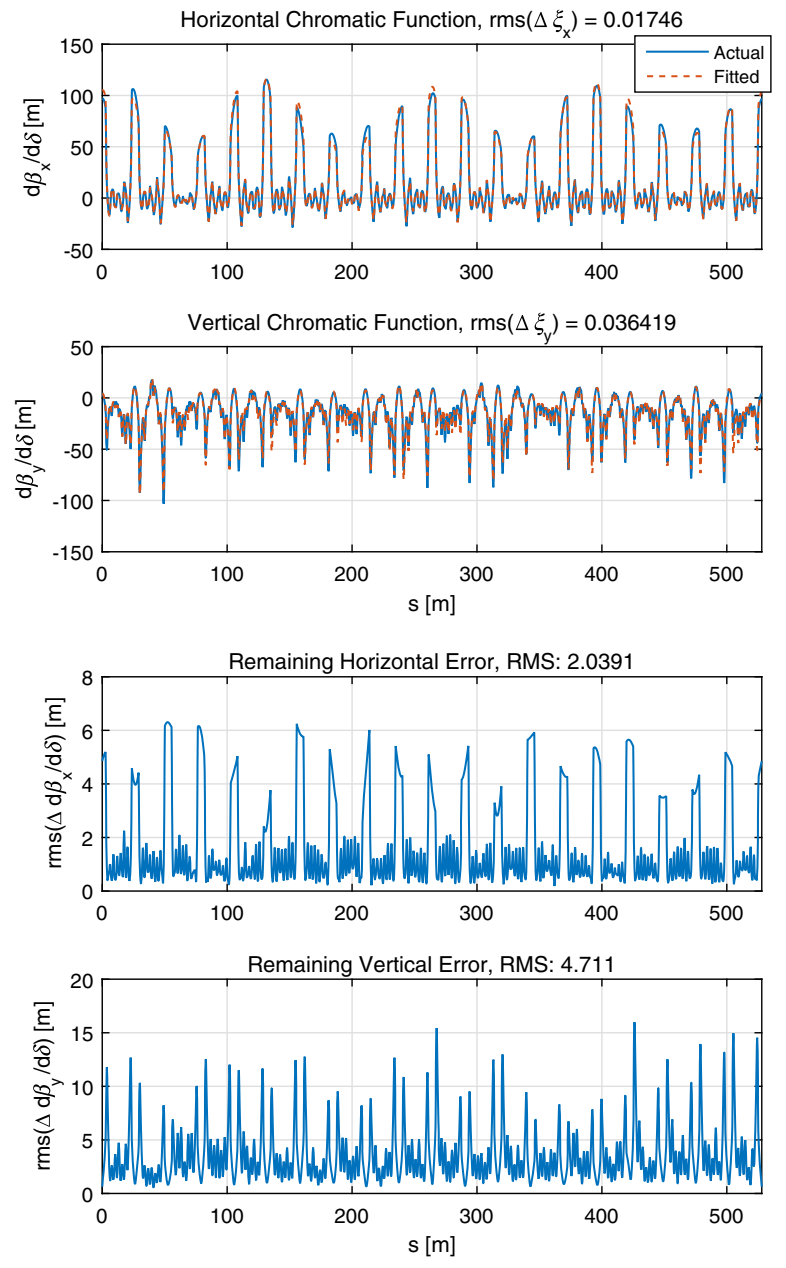

FIG. 8. The chromatic functions of one seed with sextupole magnet errors and the fitted model (top), and the RMS remaining error calculated from 10 error seeds (bottom). represented in the model as normally distributed sextupole magnet (as opposed to circuit) strength errors with the same variance and cutoff as the circuit errors. The fitting procedure is still done by circuits in order to reduce the number of fitting parameters. When allowing the fit to be done by individual sextupole magnets there are too many unknown chromatic gradients between each BPM and dipole corrector, resulting in a large number of singularities and unrealistic suggested sextupole corrections. When fitting by circuit, the magnet errors are clearly outside the parameter space of the fitting procedure, thus it cannot clearly identify the errors as in the case of the circuit errors. This can be seen in Fig. 7 where the found settings do not directly correspond to the introduced errors. Despite this the fit is able to reproduce the chromatic functions and the chromaticities (see Fig. 8 and Table II). Comparing the two situations it is clear that the fit to the circuits errors is more accurate, which is not surprising since the circuits errors are fully within the parameter space of a correction by circuit. Additionally, the chromatic function beating introduced by the magnet errors is a factor of $\sim 2$ larger than those introduced by the circuit errors. The method finds the sextupole circuit settings which closest approximates the effect of the introduced sextupole magnet errors. These are strong indications that a fit by sextupole circuit is sufficient even in the case of individual magnet errors.

\section{MEASUREMENTS}

The following measurements were performed on the MAX IV $3 \mathrm{GeV}$ storage ring. Each OEORM measurement takes approximately twice as long as a single ORM measurement, with some extra time requirements from the additional orbit correction. The measurement time scales with the number of dipole corrector magnets, BPM readback time, and dipole corrector ramp time. For example, a full OEORM took $2 \mathrm{~h}$ to measure on the MAX IV $3 \mathrm{GeV}$ storage ring without any attempts to optimize the measurement time.

Initial measurements were a proof of principle in which a number of sextupole errors were intentionally introduced to the lattice. These were identified using the NOECO scheme. The later measurements were a symmetrization of the sextupole strengths of the ring through iterative application of the correction scheme. These measurements can be seen as the sextupole equivalent of correcting the quadrupole fields in the ring using the iterative application of the LOCO scheme.

\section{A. Initial measurements}

The viability of the method was tested by investigating its ability to detect known sextupole errors in the machine. The known errors consisted of reducing the current of five different sextupole circuits by 20\%,15\%,10\%, 5\%, and $2 \%$ from nominal settings. An OEORM measurement was 

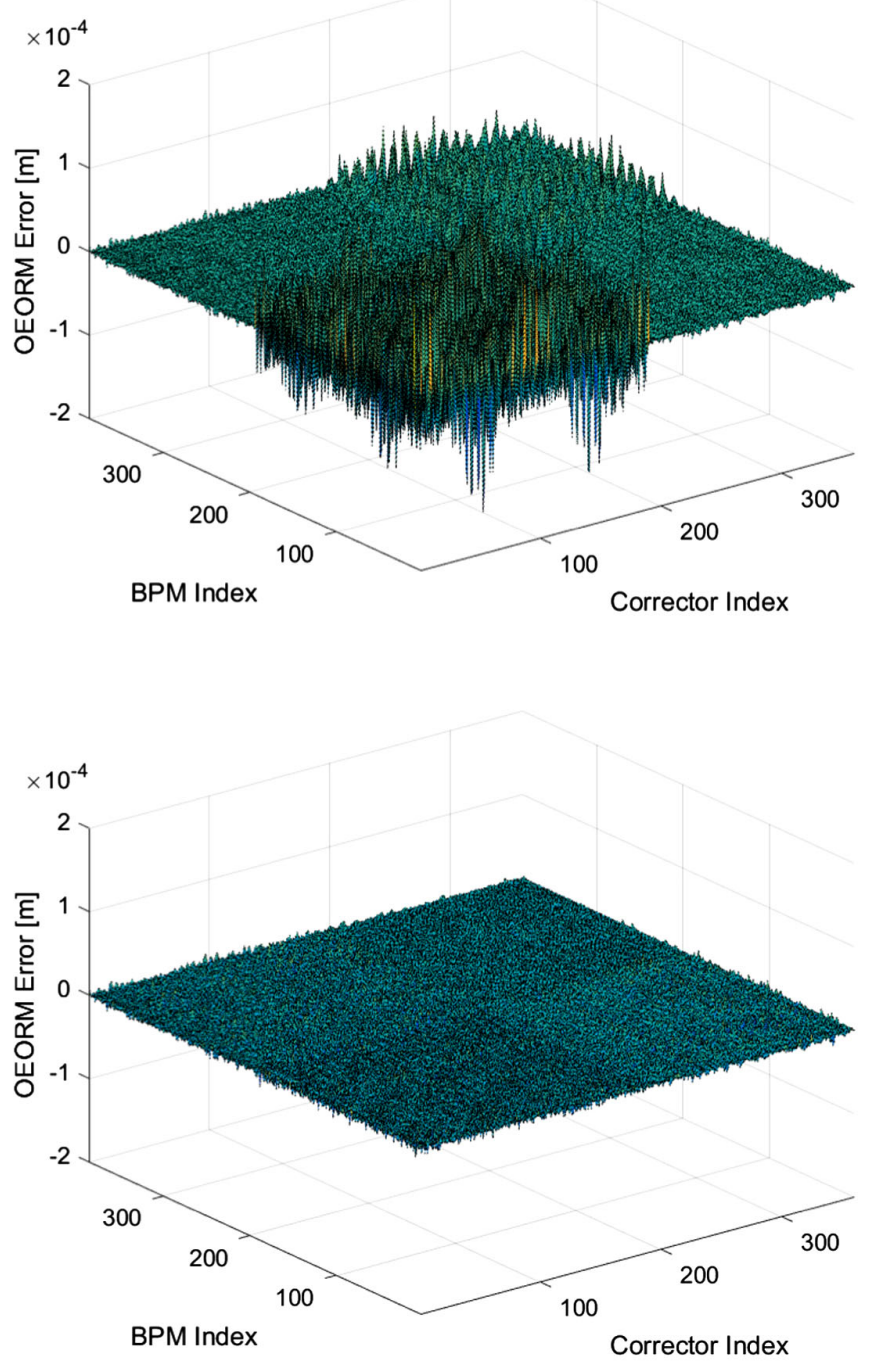

FIG. 9. Difference between measured and model OEORM before (top) and after (bottom) fitting the model. The BPMs and dipole correctors are plotted in the order horizontal followed by vertical.

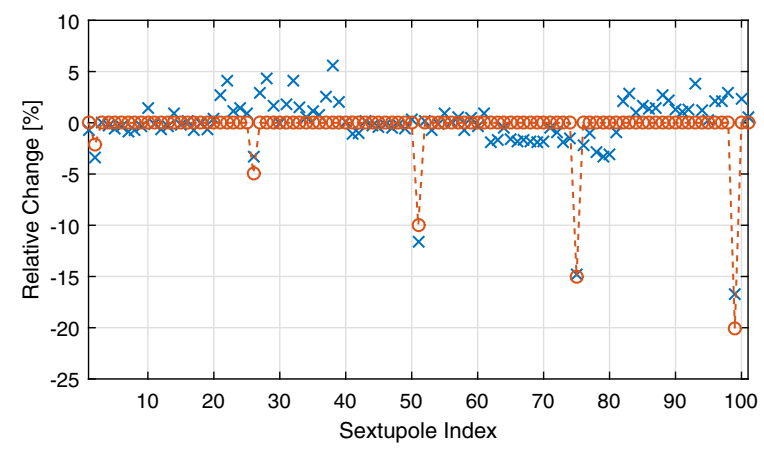

FIG. 10. Identified and introduced change in sextupole circuit strengths. The fitting procedure is able to identify all introduced errors, but a spread of errors not corresponding to the introduced errors can also be seen.
TABLE III. Measured chromaticity and chromaticity of model fitted to measured OEORM. "Before errors" refers to the machine at nominal sextupole settings, while "After errors" refers to the reductions described in Sec. VA.

\begin{tabular}{lcc}
\hline \hline & Measured $\xi_{x} / \xi_{y}$ & Fitted $\xi_{x} / \xi_{y}$ \\
\hline Before errors & $+1.1200 /+0.8531$ & $+1.0169 /+0.9889$ \\
After errors & $+0.5342 /+1.3590$ & $+0.5047 /+1.6117$ \\
\hline \hline
\end{tabular}

performed on the machine before and after applying the changes to the five sextupole circuits. A bipolar momentum shift of $\bar{\delta} \approx 1.6 \%$ and a dipole corrector kick of $\sim 0.05 \mathrm{mrad}$ was used for the measurement. The energy-shift was increased, compared to the simulations, which was shown in Sec. III to increase the signal to noise ratio. The increase drowned out the signal from a thermal drift discussed later in this section. Since this energy-shift constitutes a large beam displacement the BPM readings were linearized using the procedure found in [15]. The difference between the measured and model OEORM before and after the fitting procedure can be seen in Fig. 9 .

From the fits to the data measured before and after introducing the sextupole circuit errors, two sets of sextupole settings were found. The difference between the two settings can be seen in Fig. 10. All of the introduced errors could be identified by the fit, but a spread of errors not corresponding to the introduced errors can also be seen.

The chromaticity of the machine before and after introducing the five reductions to some sextupole circuits, along with the chromaticity of the models fitted to the measured data of the two sextupole settings, can be seen in Table III. The fitted model appears to be able to reproduce the measured chromaticity with decent accuracy.

The BPM gains and dipole corrector magnet kick strengths were also part of the fitting procedure. The fitted gains and kicks are able to identify larger gain discrepancies, e.g., the BPMs in the beginning and the end of the first achromat. These have a different gain due to having a different geometry compared to the remaining BPMs in the ring. The found BPM gains and corrector kicks can be seen in Fig. 11.

The accuracy of the fits to a measured OEORM was limited by a thermal drift of the beam position after magnet cycling. The issue was initially resolved by waiting for the machine to thermalize. Since sufficient thermalization took several hours to achieve the signal from the thermal drift was instead drowned out by increasing the energy-shift used to measured the OEORM. Additionally, periodic orbit correction was added to the OEORM measurement procedure. This reduces the effect of thermal drifts as well as corrector magnet hysteresis.

\section{B. Iterative symmetrization}

The magnitude of the sextupole corrections requested by the fits in Sec. VA was outside the range of the SDend 

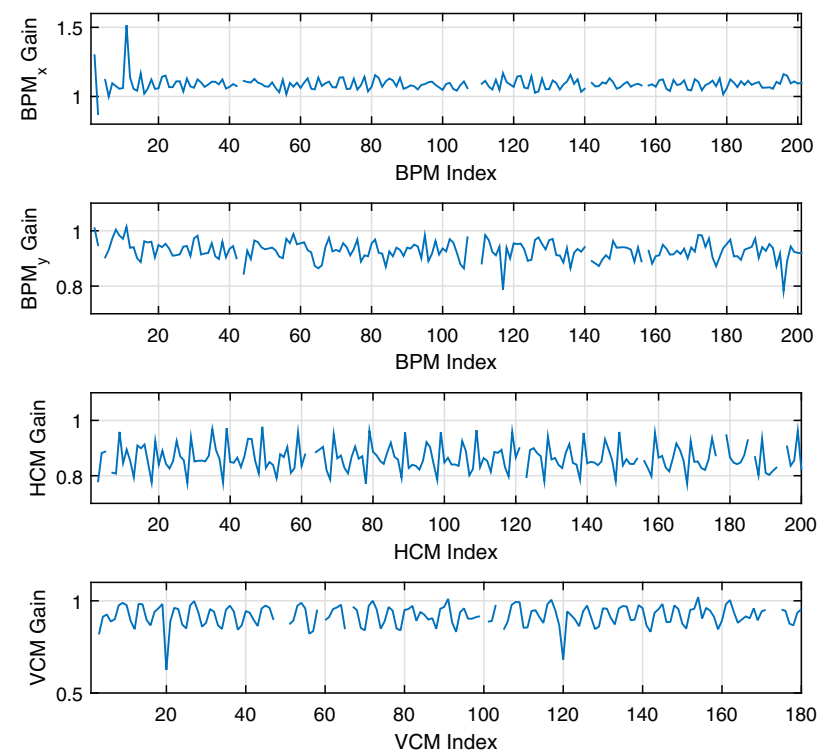

FIG. 11. BPM and dipole corrector magnet gains found by fitting to the OEORM. The method is able to identify the different gain of the flanking BPMs in the first achromat. In the case of the BPMs the "1" corresponds to the theoretical gain of a standard MAX IV $3 \mathrm{GeV}$ storage ring BPM, while in the case of the correctors 1 corresponds to the kick strength given by magnetic measurements.

family of sextupoles. The large setpoint change was due to the fitting procedure lacking measurement points (consisting of BPMs and dipole corrector magnets) between the SDend and SFm magnets. This resulted in the method increasing the strengths of the SDend and adjacent SFm sextupoles against each other. The singularity meant that large set point changes could be done to the SDend and

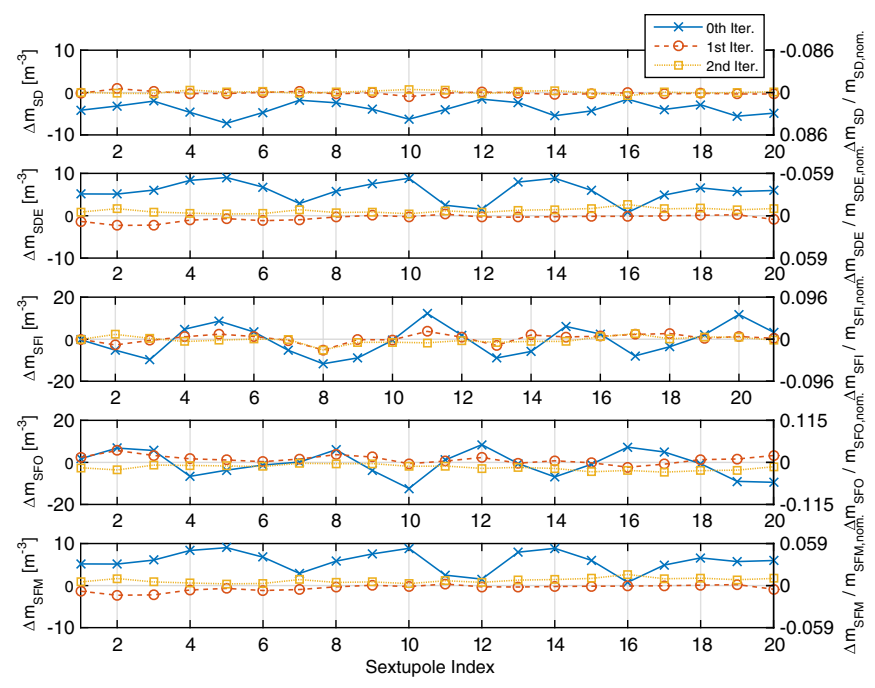

FIG. 12. Difference in sextupole strength from nominal identified from the measured OEORM in each iteration. The changes of the SDend and SFm families are the same since the circuits have been combined when fitting.
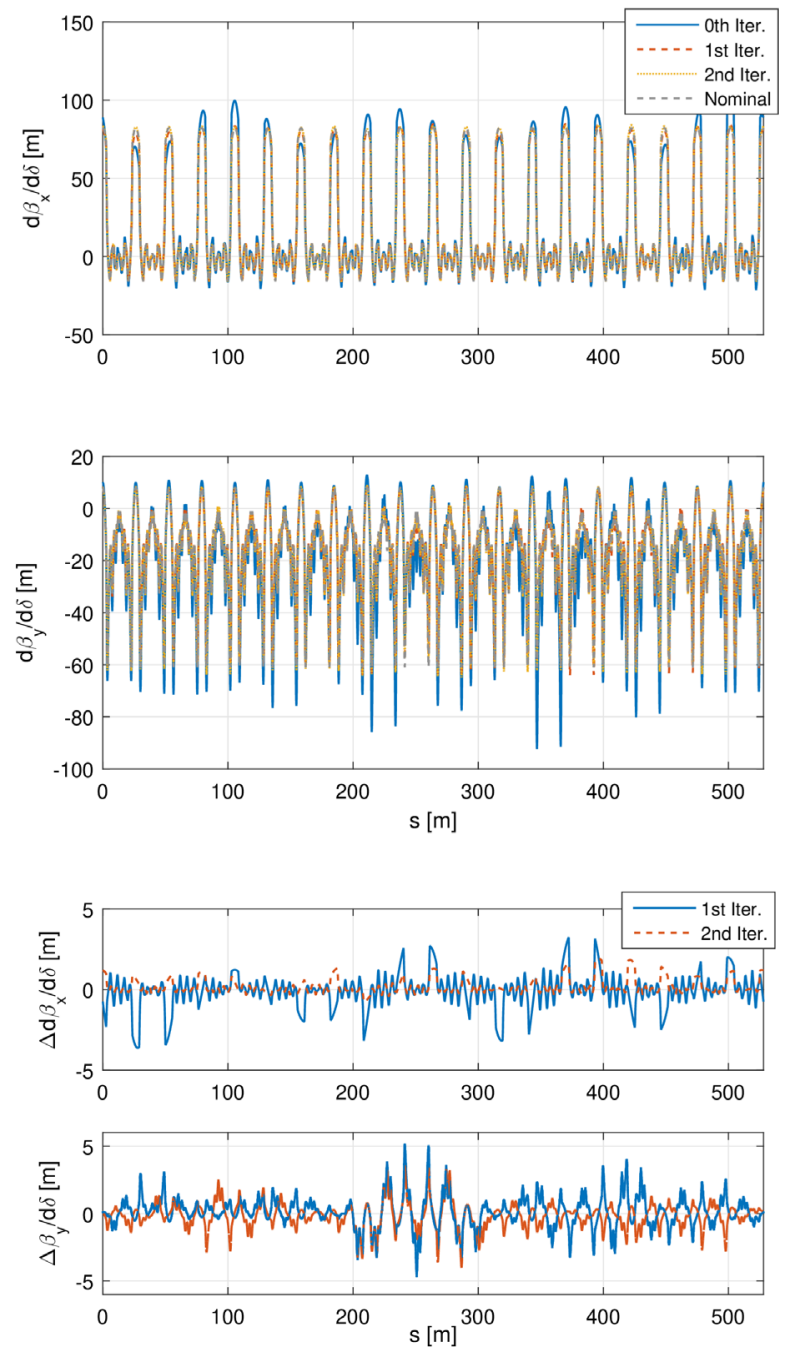

FIG. 13. Chromatic functions of the ring with each iteration of corrections. The chromatic functions were extracted from the fitted model.

SFm families without significantly affecting the OEORM residual. The issue was resolved by combining the SDend and SFm circuits for each achromat in the fitting procedure.

Prior to the iterative symmetrization the sextupole settings were returned to the theoretically nominal values according to the magnet calibration data. The linear optics were corrected using the LOCO algorithm. The OEORM was measured with a bipolar momentum shift of $\bar{\delta} \approx 1.6 \%$, and a bipolar orbit response from a kick of $\sim 0.05 \mathrm{mrad}$. The BPM readings were once again linearized using the procedure found in [15].

The initial chromaticity and the resulting chromaticity as each iteration of corrections was applied to the machine can be seen in Table IV. Figure 12 shows the identified difference in sextupole strength from nominal for each iteration. It is clear that the sextupole strength identified from the OEORM converges to the nominal settings. After only two iterations the measured chromaticity converged to 
TABLE IV. Measured chromaticity and chromaticity of model fitted to measured OEORM. The chromaticity converges to the goal values of $+1 /+1$ after two iterations.

\begin{tabular}{lcc}
\hline \hline & Measured $\xi_{x} / \xi_{y}$ & Fitted $\xi_{x} / \xi_{y}$ \\
\hline Oth iteration & $+0.9233 /+3.2345$ & $+0.7873 /+3.2507$ \\
1st iteration & $+1.2167 /+0.8254$ & $+1.1884 /+0.9677$ \\
2nd iteration & $+1.0089 /+0.9722$ & $+0.9963 /+0.9948$ \\
\hline \hline
\end{tabular}

the goal of $+1 /+1$ and the fitting procedure no longer identifies any large differences in the sextupole strengths from nominal. From the convergence of the chromaticity it appears that the iterative application of the procedure results in a higher accuracy in the predicted chromaticity than the single measurements shown in Sec. VA. This is likely due to the static Jacobian becoming more relevant as the 2nd order optics of the machine approaches nominal.

From the fitted models it is possible to extract the chromatic functions. As the sextupoles converge to the
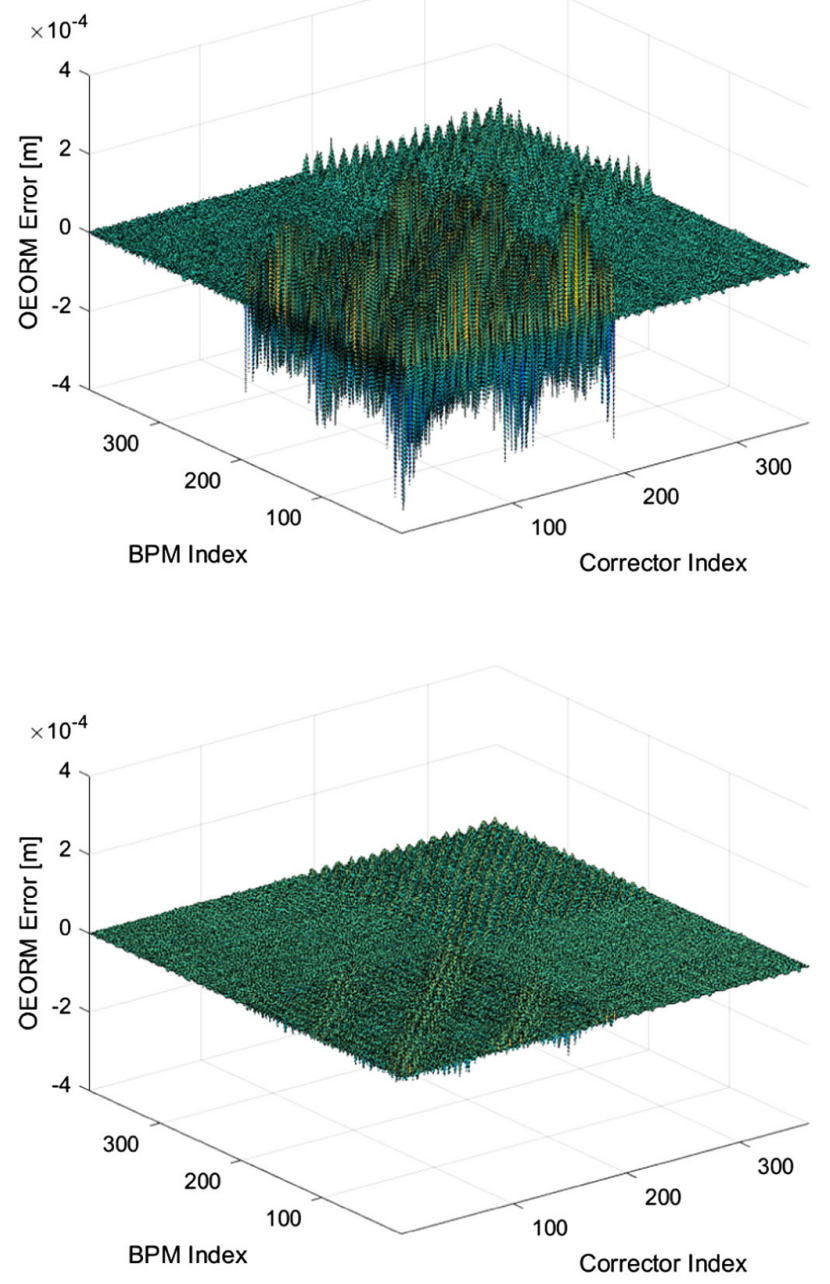

FIG. 14. Difference between measured and nominal OEORM before (top) and after (bottom) applying the sextupole symmetrization to the ring. symmetrized settings the chromatic functions converge to the nominal model values (see Fig. 13). It is worth noting that the convergence of the chromatic functions only proves that the fitting procedure is self-consistent. It is still possible that there is a chromatic function error due to the scheme not being able to perfectly reproduce the machine functions (as seen in Sec. IV). However, the predicted chromaticity values of the final iteration in Table IV match the measured very well. This is an indication that the chromatic functions are also well matched.

The convergence of the iteration process can also be investigated by looking at the difference between the measured and the nominal OEORM before and after the application of the correction. In Fig. 14 a reduction of the OEORM error by a factor of 5 can be seen. Some systematic errors in both the horizontal and the vertical plane of the matrix can still be seen.

\section{EFFECT ON DYNAMIC APERTURE}

The sextupole settings found in the iterative process of Sec. V B were applied to the ring. These settings will be referred to as the post-symmetrization settings. Their effect on the ring's dynamic aperture was investigated using three different independent measurements, namely: lifetimescraper, pinger, and momentum acceptance measurements. A lattice with the same dipole, quadrupole, and octupole settings, but with sextupoles set at their design value according to their measured magnetic field excitation curves, is used as a reference lattice when comparing and evaluating the effect on the dynamic aperture. These settings will be referred to as the presymmetrization settings. Due to the reference lattice, the presymmetrization settings, having higher coupling it had a larger vertical emittance by a factor of $\sim 5$ compared to the post-symmetrization lattice. Once the emittance coupling of the new settings had been increased to the level of the previously used settings the lifetime at delivery was $\sim 19 \mathrm{~h}$, compared to $\sim 11 \mathrm{~h}$ for the previously used sextupole settings.

\section{A. Lifetime-scraper measurements}

Lifetime-scraper measurements were performed by monitoring the beam lifetime, $\tau$, and current, $I$, while moving either a horizontal or vertical scraper closer to the beam centre. The distance from the beam center at which the scraper starts affecting the lifetime $\cdot$ current product, $I \cdot \tau$, is used as a proxy for the dynamic aperture, either horizontally or vertically. This product was used, as opposed to the lifetime directly, to compensate for the current lost during the measurements. The position where the scraper starts affecting $I \cdot \tau$ was calculated by finding the first scraper position where the $I \cdot \tau$ is statistically smaller than the initial value with a 95\% confidence interval. A beam current of $3 \mathrm{~mA}$ was used during the measurement. A short 

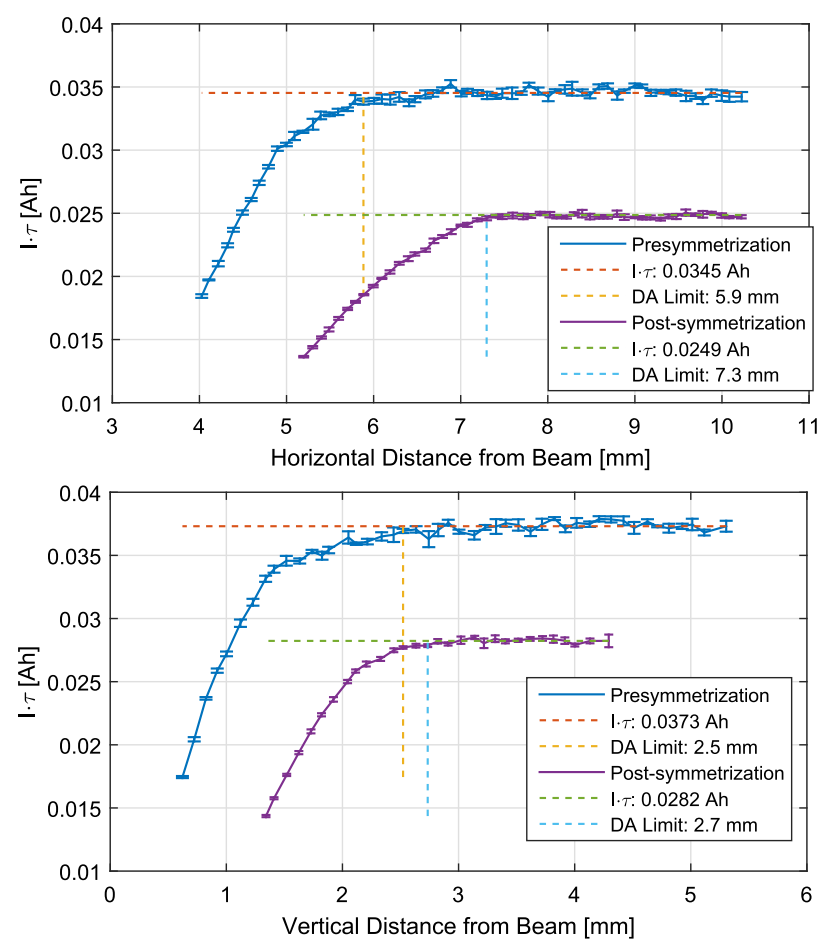

FIG. 15. $I \cdot \tau$ versus horizontal (top) and vertical (bottom) scraper distance from beam center. "Presymmetrization" refers to sextupole settings based solely on rotating coil calibration curves, while "post-symmetrization" refers to the sextupole settings found in Sec. VB. The post-symmetrization settings resulted in an increase in the horizontal dynamic aperture. The measurements were done at a beam current of $3 \mathrm{~mA}$, and a short bunch train. The difference in lifetime was due to the lower vertical emittance from a lower coupling in the post-symmetrization lattice.

bunch train was used to ensure that the lifetime was low enough to be measured both accurately and quickly.

The post-symmetrization settings resulted in an increase in the horizontal dynamic aperture from $5.9 \pm 0.2 \mathrm{~mm}$ to $7.3 \pm 0.2 \mathrm{~mm}$, and in the vertical from $2.5 \pm 0.2 \mathrm{~mm}$ to $2.7 \pm 0.2 \mathrm{~mm}$ (see Fig. 15). The corresponding transverse acceptance values are $3.7 \pm 0.1 \mathrm{~mm} \mathrm{mrad}$ and $5.6 \pm$ $0.2 \mathrm{~mm}$ mrad horizontally, and $1.6 \pm 0.2 \mathrm{~mm} \mathrm{mrad}$ and $1.9 \pm 0.2 \mathrm{~mm}$ mrad vertically. It is possible that the horizontal scraper limits the momentum acceptance as well as the horizontal acceptance, since a scraper at a nondispersive section can still catch a Touschek scattered particle. If this were the case, the scraper measurement would show that the post-symmetrization sextupole settings result in an increase in momentum acceptance. In order to more directly measure the acceptances additional dipole pinger and $\mathrm{rf}$ momentum variation measurements were performed.

\section{B. Pinger measurements}

The horizontal and vertical dipole pinger magnets of the MAX IV $3 \mathrm{GeV}$ storage ring were used to measure
TABLE V. Measured horizontal and vertical acceptance of the presymmetrization and post-symmetrization sextupole settings.

\begin{tabular}{lcc}
\hline \hline & $A_{x}[\mathrm{~mm} \mathrm{mrad}]$ & $A_{y}[\mathrm{~mm} \mathrm{mrad}]$ \\
\hline Presymmetrization & $2.1 \pm 0.1$ & $2.1 \pm 0.1$ \\
Post-symmetrization & $3.9 \pm 0.1$ & $1.2 \pm 0.1$ \\
\hline \hline
\end{tabular}

the transverse acceptance of the beam while using either the post-symmetrization sextupole settings or the presymmetrization settings. A stored beam of $3 \mathrm{~mA}$ was kicked in one plane with an iteratively increasing kick strength until at least $1 \%$ of the stored beam is lost with each kick. A short bunch train was used in order to ensure that all bunches see the same dipole kick amplitude. The value of the maximum kick was taken as the limit of the beam kick resilience in that plane. The $1 \%$ limit was chosen in order to save time during the measurement, and avoid frequent beam injections.

The resulting transverse acceptances calculated from the kick resiliences of the two different sextupole settings can be seen in Table V. The horizontal acceptance was increased by the post-symmetrization sextupole settings, while the vertical was decreased. Since only a $1 \%$ beam loss was allowed during the measurement these values are a lower limit of the actual transverse acceptances, but are still useful for measuring changes in acceptance. In order to get a more precise acceptance measurement a beam loss of $50 \%$ would be more appropriate. A good explanation to why the vertical scraper and pinger measurements give different results is yet to be found. However, this ambiguity will have a minor influence, since during user delivery the vertical physical acceptance is limited to $1 \mathrm{~mm} \mathrm{mrad}$ by the IDs.

\section{Momentum acceptance measurements}

The momentum acceptances of the two lattices were measured by monitoring the lifetime while changing the accelerating voltage of the cavities. A short bunch train was used to reduce multibunch effects. The beam was kept stable throughout the measurement. From OPA [16] a theoretical lifetime-accelerating voltage curve depending only on the rf-acceptance was calculated. The accelerating voltage could be transformed into momentum acceptance via the synchrotron frequency which was also measured during the experiment. When any part of the studied lattices start to limit the momentum acceptance, the corresponding measured lifetime will start to deviate from the theoretical lifetime. As more parts of the lattice limits the momentum acceptance the curves will diverge more. In Fig. 16 the ratio between the measured and the theoretical Touschek lifetime can be seen. At our delivery rf momentum acceptance of $5.4 \%$, the Touschek lifetime increased a factor of 2 , due to the improved lattice momentum acceptance.

The improvement of the momentum acceptance can also be seen from the models fitted to the initial and final 


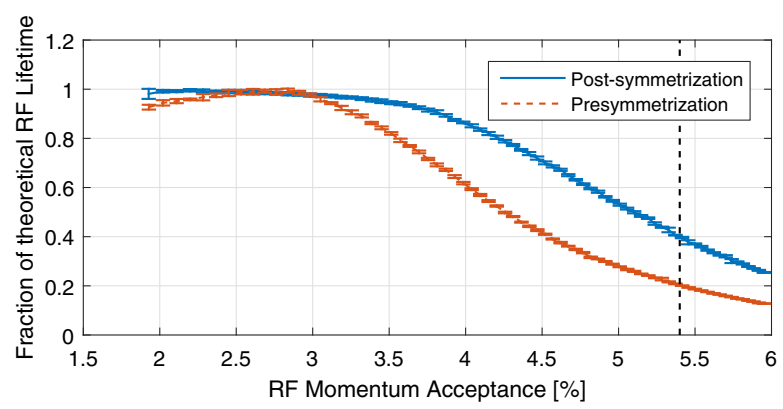

FIG. 16. Measured lifetime plotted against rf momentum acceptance as a ratio of measured lifetime to simulated lifetime only determined by the rf-acceptance. Both the measured and simulated lifetimes were normalized against their maxima before taking the ratio. The vertical dashed line corresponds to the rf momentum acceptance of the MAX IV $3 \mathrm{GeV}$ ring during delivery. At this acceptance value the symmetrization increased the Touschek lifetime by a factor of 2 .

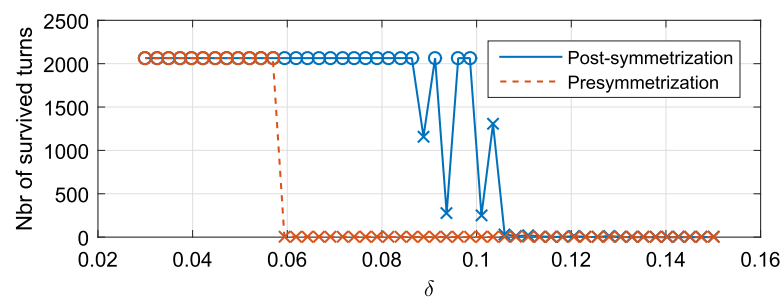

FIG. 17. Number of survived turns for a particle with momentum deviation $\delta$ propagating in the model fitted to the pre- and post-symmetrization OEORM measurements. A momentum acceptance increase of $\sim 50 \%$ can be seen. The simulation was run for 2064 turns.

OEORM measurements. The momentum acceptance limit was probed by simulating a particle with a certain energy deviation $\delta$ for 2064 turns, corresponding to $>4$ full synchrotron periods. Losing the particle indicates the limit of the acceptance. From the results of the simulation (see Fig. 17) it is clear that the increase in momentum acceptance is present also in the model.

\section{CONCLUSIONS}

The novel NOECO scheme, described in this paper, was applied on the MAX IV $3 \mathrm{GeV}$ storage ring lattice. The scheme symmetrized the chromatic functions and sextupole settings through fitting of a lattice model to measured offenergy orbit response matrices. It was able to qualitatively and quantitatively, down to single percent level, identify sextupole errors intentionally introduced to the lattice. Through iterative corrections the method was also able to converge on a new set of sextupole settings, which in turn resulted in the chromaticity converging to the goal values. The new sextupole setting proved to be an improvement of $\sim 30-40 \%$ with regards to horizontal dynamic acceptance, as measured by the pinger acceptance measurement. The settings also showed a factor of 2 increase of the Touschek lifetime at the operating rf acceptance of the ring. The new sextupole settings are currently in use during delivery at the MAX IV $3 \mathrm{GeV}$ storage ring. The emittance coupling of the new optics has been increased to the level previously used during delivery. The resulting lifetime of the new settings at delivery conditions is $\sim 19 \mathrm{~h}$, compared to $\sim 11 \mathrm{~h}$ for the previously used sextupole settings.

The fitting and symmetrization of the MAX IV $3 \mathrm{GeV}$ sextupoles was done by magnet circuit, rather than by individual magnets. This was due to there being several individual sextupoles between the measurement points (BPMs and dipole correctors) of the MAX IV $3 \mathrm{GeV}$ ring lattice. The NOECO scheme was not able to separate these individual gradients as these singularities instead had a tendency to increase the strength of adjacent gradients against each other. This resulted in unrealistic sextupole settings which were too large to be implemented. The same issue arose when fitting by circuits as the SDend and SFm sextupoles lack a measurement point between each other. This was resolved by combining the SDend and SFm families, at which point the sextupole circuits were sufficient fitting parameters to symmetrize the 2 nd order lattice.

The accuracy of the NOECO scheme, when applied to the MAX IV $3 \mathrm{GeV}$ storage ring, was limited by the thermal drift of the machine after magnet cycling, and by BPM noise. The effect of the thermal drift could be reduced without waiting for thermalization by increasing the energy-shift at which the OEORMs are measured. The effect was also decreased by correcting the beam position periodically throughout the measurement. In the case of the MAX IV $3 \mathrm{GeV}$ storage ring this was a sufficient reduction to unwanted signals during the measurement. Since noise and drifts are machine dependent the optimal energy-shift and amount of orbit correction will be different for each machine.

Harmonic sextupoles are completely transparent to the NOECO scheme and are thus not able to be symmetrized by the scheme. This is not an issue in the MAX IV $3 \mathrm{GeV}$ ring as all its sextupoles are chromatic. If the scheme is to be used on a lattice with harmonic sextupoles it will need to be complemented with other methods. Higher order magnets, such as octupoles, have a minuscule effect on the OEORM and could therefore safely be excluded from the fitting procedure without affecting the final sextupole symmetrization. The octupole magnets of the MAX IV $3 \mathrm{GeV}$ storage ring play an important role in the amplitude dependent tune-shift and the transverse dynamic acceptance of the ring. However, the choice was made to limit this paper to sextupoles in order to investigate one order of nonlinear magnets at a time. Since the octupole magnets of the MAX IV $3 \mathrm{GeV}$ storage ring are powered globally they constitute a very limited parameter space, and are planned to be characterized and corrected through the amplitude dependent tune-shift. 
The novel NOECO scheme has the potential to be used for the symmetrization of the chromatic sextupoles of not only the MAX IV $3 \mathrm{GeV}$ storage ring but for many other fourth generation storage rings, provided that the BPM and dipole corrector magnet layout sufficiently samples the chromatic sextupole lattice. The method provides a structured approach to identifying all chromatic sextupole gradients of the ring from a single measurement which is no more time consuming than two ORM measurements.

\section{ACKNOWLEDGMENTS}

We would like to thank Pedro Fernandes Tavares for the fruitful discussions and his insightful comments.

[1] R. Bartolini, I. P. S. Martin, G. Rehm, and F. Schmidt, Calibration of the nonlinear ring model at the Diamond Light Source, Accel. Beams Phys. Rev. 14, 054003 (2011).

[2] I. Agapov, M. Bieler, H. Ehrlichmann, J. Keil, J. Klute, G. Kube, G. K. Sahoo, F. Schmidt-Foehre, R. Wanzenberg, R. Tomás, and A. Wegscheider, Linear and nonlinear optics analysis from multiturn data at PETRA III, Proc. 8th Int. Accelerator Conf. (IPAC2017), Copenhagen, Denmark (2017), pp. 170-173, http://accelconf.web.cern .ch/ipac2017/papers/mopab040.pdf.

[3] J. Bengtsson, I. P. S. Martin, J. H. Rowland, and R. Bartolini, On-line control of the nonlinear dynamics for synchrotrons, Accel. Beams Phys. Rev. 18, 074002 (2015).

[4] J. Safranek, Experimental determination of storage ring optics using orbit response measurements, Nucl. Instrum. Methods Phys. Res. 388, 27 (1997).

[5] P. F. Tavares, E. Al-Dmour, A. Andersson, F. Cullinan, B. N. Jensen, D. Olsson, D. K. Olsson, M. Sjöström, H. Tarawneh, S. Thorin, and A. Vorozhtsov, Commissioning and first-year operational results of the MAX IV $3 \mathrm{GeV}$ ring, J. Synchrotron Radiat. 25, 1291 (2018).
[6] D. K. Olsson, A. Andersson, and M. Sjöström, 2nd order optics symmetrisation through off-energy orbit response matrix, Proc. 10th Int. Accelerator Conf., Melbourne, Australia (IPAC2019) (2019), pp. 841-843, http:// accelconf.web.cern.ch/ipac2019/papers/mopts004.pdf.

[7] N. Carmignani, Sextupole calibrations via measurements of off-energy orbit response matrix and high order dispersion, presented at the 25th European Synchrotron Light Source Workshop (ESLS'17), Dortmund, Germany, Nov. 2017, https://indico.cern.ch/event/657829/contributions/2782617/ attachments/1569843/2475779/ESLS17_Carmignani_ SextCalibration.pdf.

[8] Detailed Design Report, Technical report, MAX IV Laboratory, 2010.

[9] G. Portmann, J. Corbett, and A. Terebilo, An accelerator control middle layer using MATLAB, in Proceedings of the 21st Particle Accelerator Conference, Knoxville, TN, 2005 (IEEE, Piscataway, NJ, 2005).

[10] X. Huang, Robust simplex algorithm for online optimization, Accel. Beams Phys. Rev. 21, 104601 (2018).

[11] D. K. Olsson, Online Optimisation of the MAX IV $3 \mathrm{GeV}$ Ring Dynamic Aperture, Proc. 9th Int. Accelerator Conf., Vancouver, Canada (IPAC2018) (2018), pp. 2281-2283, http://accelconf.web.cern.ch/ipac2018/papers/wepal047 .pdf.

[12] H. Wiedemann, Particle Accelerator Physics, 3rd ed. (Springer, New York, 2007), chap. 14, pp. 503-534.

[13] X. Huang, The dipole pass method for Accelerator Toolbox, Accelerator Physics Note 021, SLAC National Accelerator Laboratory, 2009.

[14] A. Terebilo, Accelerator modeling with MATLAB Accelerator Toolbox, in Proceedings of the 19th Particle Accelerator Conference, Chicago, IL, 2001 (IEEE, Piscataway, NJ, 2001).

[15] A. Stella, Analysis of the DAFNE beam position monitor with a boundary element method, DAFNE Technical Note CD-10, INFN-LNF, Accelerator Division, 1997.

[16] OPA lattice design code. ados.web.psi.ch/opa/, 2012. 